\title{
UNIQUE SOLUTION TO PERIODIC BOUNDARY VALUE PROBLEMS ${ }^{1}$
}

\author{
YONG SUN \\ Department of Applied Mathematics \\ Florida Institute of Technology \\ Melbourne, FL 32901
}

\begin{abstract}
Existence of unique solution to periodic boundary value problems of differential equations with continuous or discontinuous righthand side is considered by utilizing the method of lower and upper solutions and the monotone properties of the operator. This is subject to discussion in the present paper.
\end{abstract}

Key words: existence and uniqueness of solution, differential equations, lower and upper solutions.

AMS (MOS) subject classifications: 47H, 34B.

\section{INTRODUCTION}

In this paper, we utilize the method of lower and upper solutions and the monotone properties of the operator and study the existence and uniqueness of solutions of periodic boundary value problem for first order differential equations. In general, we assume that the upper solution dominates the lower solution. However, it is interesting and valuable to study a problem when the lower solution dominates the upper solution. We discuss both continuous and discontinuous cases.

\footnotetext{
${ }^{1}$ Received: December 1990, Revised: April 1991
} 


\section{CONTINUOUS RIGHT-HAND SIDE}

Consider the first order periodic boundary value problem

$$
\begin{aligned}
& u^{\prime}=f(t, u), t \in J ; \\
& u(0)=u(2 \pi),
\end{aligned}
$$

where $\mathrm{J}=[0,2 \pi]$. A function $\alpha \in C^{1}([0,2 \pi], R)$ is said to be a lower solution of $\operatorname{PBVP}(2.1),(2.2)$ if $\alpha^{\prime} \leq f(t, \alpha)-\gamma_{\alpha}$, where

$$
\gamma_{\alpha}= \begin{cases}0 & \text { if } \alpha(0) \leq \alpha(2 \pi), \\ M[\alpha(0)-\alpha(2 \pi)] \frac{e^{2 M \pi}}{e^{2 M \pi}-1} & \text { if } \alpha(0)>\alpha(2 \pi)\end{cases}
$$

And a function $\beta \in C^{1}([0,2 \pi], R)$ is said to be an upper solution of PBVP (2.1), (2.2) if $\beta^{\prime} \geq f(t, \beta)+\gamma_{\beta}$, where

$$
\gamma_{\beta}= \begin{cases}0 & \text { if } \beta(0) \geq \beta(2 \pi), \\ M[\beta(2 \pi)-\beta(0)] \frac{e^{2 M \pi}}{e^{2 M \pi}-1} & \text { if } \beta(0)<\beta(2 \pi)\end{cases}
$$

For the sake of convenience we recall a result about differential inequality in $[2,3]$.

Lemma 2.1. Let $m \in C^{1}([0,2 \pi], R)$ and $m^{\prime} \geq M m+\gamma_{m}$ where

$$
\gamma_{m}= \begin{cases}0 & \text { if } m(0) \geq m(2 \pi) \\ M[m(2 \pi)-m(0)] \frac{e^{2 M \pi}}{e^{2 M \pi}-1} & \text { if } m(0)<m(2 \pi)\end{cases}
$$

where $M>0$. Then $m(t) \leq 0$ on $[0,2 \pi]$.

We now present the main result of this section.

Theorem 1. Let $\alpha, \beta \in C^{1}([0,2 \pi], R)$ be the lower and upper solutions to (2.1)-(2.2) and $\beta \leq \alpha$. Suppose that $\mathrm{f}: J \times R \rightarrow R$ is continuous and 


$$
f(t, x)-f(t, y) \leq M(x-y) \text { whenever } \beta(t) \leq y \leq x \leq \alpha(t),
$$

where $M>0$. Then PBVP (2.1)-(2.2) possesses a unique solution in the sector $[\beta, \alpha]$.

Proof. For any $v \in[\beta, \alpha]$, we consider the linear PBVP

$$
u^{\prime}-M u=f(t, v(t))-M v(t), \quad u(0)=u(2 \pi) .
$$

Setting $F(t, x)=f(t, x)-M x$ for $(t, x) \in J \times R$. Then it is easy to verify that

$$
\begin{aligned}
& u(t)=u(0) e^{M t}+\int_{0}^{t} F(s, v(s)) e^{M(t-s)} d s \quad \text { and } \\
& u(0)=u(2 \pi)=\frac{1}{e^{-2 M \pi}-1} \int_{0}^{2 \pi} F(s, v(s)) e^{-M s} d s,
\end{aligned}
$$

is a solution of PBVP (I). From Lemma 2.1 it follows that PBVP (I) possesses a unique solution. Therefore $\mathrm{u}(\mathrm{t})$ defined above is the unique solution of PBVP (I). And hence we define an operator $\mathrm{A}$ on the sector $[\beta, \alpha]$ by $A v=u$, where $u$ is the unique solution of PBVP (I). We now show the following two conclusions.

i) $\quad \beta \leq A \beta, \quad A \alpha \leq \alpha$

ii) $\mathrm{A}$ is increasing on the sector $[\beta, \alpha]$.

First let us show that $\mathrm{i}$ ) is true. In fact, if we set $p=\beta-A \beta$ and set

$$
\gamma_{p}= \begin{cases}0 & \text { if } p(0) \leq p(2 \pi) \\ M[p(0)-p(2 \pi)] \frac{e^{2 M \pi}}{e^{2 M \pi}-1} & \text { if } p(0)>p(2 \pi)\end{cases}
$$

Then $p(0) \leq p(2 \pi)$ if and only if $\beta(0) \leq \beta(2 \pi)$ and $p(0)>p(2 \pi)$ if and only if $\beta(0)>$ $\beta(2 \pi)$. And hence $\gamma_{p}=0$ if and only if $\beta(0) \geq \beta(2 \pi)$ and $\gamma_{p}=M[p(2 \pi)-p(0)] \frac{e^{2 M \pi}}{e^{2 M \pi}-1}$ $=M[\beta(2 \pi)-\beta(0)] \frac{e^{2 M \pi}}{e^{2 M \pi}-1}$ if and only if $\beta(0)<\beta(2 \pi)$. From this it follows that

$$
\begin{aligned}
& p^{\prime}=\beta^{\prime}-(A \beta)^{\prime} \geq f(t, \beta)+\gamma_{\beta}-M A \beta-f(t, \beta)+M \beta \\
& =M p+\gamma_{\beta}=M p+\gamma_{p} .
\end{aligned}
$$


So Lemma 2.1 implies $p \leq 0$. Thus $\beta \leq A \beta$. Similarly, we can show that $A \alpha \leq \alpha$.

We now show that $\mathrm{A}$ is increasing on the sector $[\beta, \alpha]$. In fact, if $A v_{1}=u_{1}$ and $A v_{2}=u_{2}$, where $v_{1}, v_{2} \in[\beta, \alpha]$ and $v_{1} \leq v_{2}$. Setting $\mathrm{p}=u_{1}-u_{2}$ we see that

$$
\begin{aligned}
& p^{\prime}=u_{1}^{\prime}-u_{2}^{\prime}=M u_{1}+f\left(t, v_{1}\right)-M v_{1}-M u_{2}-f\left(t, v_{2}\right)+M v_{2} \\
& \geq M p \text { and } \\
& p(0)=p(2 \pi) .
\end{aligned}
$$

This leads to $p \leq 0$ from Lemma 2.1. So $u_{1} \leq u_{2}$, and hence $\mathrm{A}$ is increasing on the sector $[\beta, \alpha]$.

On the other hand, we define an operator $B$ on the sector $[\beta, \alpha]$ as follows:

$$
\begin{aligned}
& B v(t)=B v(0) e^{M t}+\int_{0}^{t} F(s, v(s)) e^{M(t-s)} d s \quad \text { and } \\
& B v(0)=B v(2 \pi)=\frac{1}{e^{-2 M \pi}-1} \int_{0}^{2 \pi} F(s, v(s)) e^{-M s} d s
\end{aligned}
$$

Then $B$ is decreasing on the sector $[\beta, \alpha]$ since $F(t, x)$ is decreasing in $x$ from the conditions imposed on $f$. But obviously $B v(t)$ is a solution of PBVP (I). So operator $B$ is identical with operator $A$ on the sector $[\beta, \alpha]$ from the uniqueness of solution to PBVP (I). And hence operator $A$ is both increasing and decreasing on the sector $[\beta, \alpha]$ and satisfies i). Thus $A$ transforms the sector $[\beta, \alpha]$ onto a point $u^{*} \in[\beta, \alpha]$. This implies that $u^{*}$ is the unique fixed point of $A$ on the sector $[\beta, \alpha]$. However solving PBVP (2.1)-(2.2) is equivalent to finding fixed points of operator $A$. Hence $u^{*}$ is the unique solution of PBVP (2.1)-(2.2) on the sector $[\beta, \alpha]$.

Remark It is obvious that the above theorem can not be proved by applying either comparision theorem or operator theoty. So it should be noted that it is effective to combine operator theory with comparision results.

\section{DISCONTINUOUS RIGHT-HAND SIDE}

Let us consider the the following periodic boundary value problem 


$$
\begin{aligned}
& u^{\prime}=f(t, u), \text { a.e. } t \in J \\
& u(0)=u(2 \pi),
\end{aligned}
$$

where $\mathrm{J}=[0,2 \pi]$. A function $\alpha \in A C([0,2 \pi], R)$ is said to be a lower solution if $\alpha^{\prime} \leq f(t, \alpha)-\gamma_{\alpha}$ for almost all $t \in J$, where

$$
\gamma_{\alpha}= \begin{cases}0 & \text { if } \alpha(0) \leq \alpha(2 \pi), \\ M[\alpha(0)-\alpha(2 \pi)] \frac{e^{2 M \pi}}{e^{2 M \pi}-1} & \text { if } \alpha(0)>\alpha(2 \pi)\end{cases}
$$

Similarly a function $\beta \in A C([0,2 \pi], R)$ is said to be an upper solution if $\beta^{\prime} \geq$ $f(t, \beta)-\gamma_{\beta}$ for almost all $\mathrm{t}$ in $\mathrm{J}$, where

$$
\gamma_{\beta}= \begin{cases}0 & \text { if } \beta(0) \geq \beta(2 \pi), \\ M[\beta(2 \pi)-\beta(0)] \frac{e^{2 M \pi}}{e^{2 M \pi}-1} & \text { if } \beta(0)<\beta(2 \pi)\end{cases}
$$

In order to present the main result of the section we first show a result that is similar to Lemma 2.1 .

Lemma 3.1. Let $m \in A C([0,2 \pi], R)$ and $m^{\prime} \geq M m+\gamma_{m}$ for almost all $t$ in $[0,2 \pi]$, where

$$
\gamma_{m}= \begin{cases}0 & \text { if } m(0) \geq m(2 \pi), \\ M[m(2 \pi)-m(0)] \frac{e^{2 M \pi}}{e^{2 M \pi}-1} & \text { if } m(0)<m(2 \pi)\end{cases}
$$

where $M>0$. Then $m(t) \leq 0$ on $[0,2 \pi]$.

Proof. If the conclusion were not true, then $c=\sup \{m(t): t \in[0,2 \pi]\}>0$ and a $t^{*} \in[0,2 \pi]$ could be found such that $m\left(t^{*}\right)=c$. Suppose that $0 \leq t^{*}<2 \pi$. Then we see that $t_{k}>t^{*}(\mathrm{k}=1,2, \ldots)$ can be found such that $t_{k}$ tends to $t^{*}$ as $\mathrm{k}$ goes to infinity and

$$
m^{\prime}\left(t_{k}\right) \leq 0 \quad \text { and } \quad m^{\prime}\left(t_{k}\right) \geq M m\left(t_{k}\right)+\gamma_{m} .
$$

This implies that

$$
0 \geq m^{\prime}\left(t_{k}\right) \geq M m\left(t_{k}\right)+\gamma_{m} \geq \frac{1}{2} M c>0
$$


is true when $\mathrm{k}$ is sufficently large. This contradiction implies that $t^{*}$ cannot lie in $[0,2 \pi)$. So $t^{*}=2 \pi$. But this is impossible also. In fact, in this case, we can assume that $m(2 \pi)>m(0)$ without loss of generality(otherwise we take $\left.t^{*}=0\right)$. It follows from

$$
m^{\prime} \geq M m+\gamma_{m} \text { a.e. } t \in J
$$

that

$$
\left(m^{\prime}-M m\right) e^{-M t} \geq \gamma_{m} e^{-M t} \text { a.e. } t \in J
$$

Integrating the above inequality from 0 to $2 \pi$ leads to

$$
m(2 \pi) e^{-2 M \pi}-m(0) \geq-\gamma_{m}\left[e^{-2 M \pi}-1\right] / M=m(2 \pi)-m(0) .
$$

This yields that

$$
m(2 \pi)\left[1-e^{-2 M \pi}\right] \leq 0
$$

Therefore $m(2 \pi) \leq 0$. This contradicts $m(2 \pi)>0$. And hence $m(t) \leq 0$.

Theorem 2. Let $\alpha, \beta \in A C([0,2 \pi], R)$ be the lower and upper solutions to (3.1)(3.2) and $\beta \leq \alpha$. Suppose that $f: J \times R \rightarrow R$ is a function such that

i) $f(t, x(t))$ is Lebegue integrable over $\mathrm{J}$ for each $x(t)$ that lies in the sector $[\beta, \alpha]$

ii) $\quad f(t, x)-f(t, y) \leq M(x-y) \quad$ whenever $\beta(t) \leq y \leq x \leq \alpha(t)$,

where $M>0$. Then PBVP (3.1)-(3.2) possesses a unique solution in the sector $[\beta, \alpha]$.

Proof. For every $v \in[\beta, \alpha]$, we consider the following linear PBVP

$$
u^{\prime}-M u=f(t, v(t))-M v(t) \text { a.e. } \in J ; \quad u(0)=u(2 \pi) .
$$

Setting $F(t, x)=f(t, x)-M x$ for $(t, x) \in J \times R$. Then it is easy to verify that 


$$
\begin{aligned}
& u(t)=u(0) e^{M t}+\int_{0}^{t} F(s, v(s)) e^{M(t-s)} d s \quad \text { and } \\
& u(0)=u(2 \pi)=\frac{1}{e^{-2 M \pi}-1} \int_{0}^{2 \pi} F(s, v(s)) e^{-M s} d s
\end{aligned}
$$

is a solution of PBVP (II). From Lemma 3.1 it follows that PBVP (II) possesses a unique solution. Therefore $u(t)$ defined above is the unique solution of PBVP (II). Hence we define an operator $\mathrm{A}$ on uhe sector $[\beta, \alpha]$ by $A v=u$, where $u$ is the unique solution of PBVP (II). We now show the following two conclusions:

(i) $\quad \beta \leq A \beta, \quad A \alpha \leq \alpha$

(ii) $\mathrm{A}$ is increasing on the sector $[\beta, \alpha]$.

First let us show that (i) is true. In fact, if we set $p=\beta-A \beta$ and set

$$
\gamma_{p}= \begin{cases}0 & \text { if } p(0) \leq p(2 \pi) \\ M[p(0)-p(2 \pi)] \frac{e^{2 M \pi}}{e^{2 M \pi}-1} & \text { if } p(0)>p(2 \pi)\end{cases}
$$

Then $p(0) \leq p(2 \pi)$ if and only if $\beta(0) \leq \beta(2 \pi)$ and $p(0)>p(2 \pi)$ if and only if $\beta(0)>$ $\beta(2 \pi)$. And hence $\gamma_{p}=0$ if and only if $\beta(0) \geq \beta(2 \pi)$ and $\gamma_{p}=M[p(2 \pi)-p(0)] \frac{e^{2 M \pi}}{e^{2 M \pi}-1}$ $=M[\beta(2 \pi)-\beta(0)] \frac{e^{2 M \pi}}{e^{2 M \pi}-1}$ if and only if $\beta(0)<\beta(2 \pi)$. From this it follows that

$$
\begin{aligned}
& p^{\prime}=\beta^{\prime}-(A \beta)^{\prime} \geq f(t, \beta)+\gamma_{\beta}-M A \beta-f(t, \beta)+M \beta \\
& =M p+\gamma_{\beta}=M p+\gamma_{p} .
\end{aligned}
$$

So Lemma 3.1 shows that $p \leq 0$. This implies that $\beta \leq A \beta$. Similarly, we can show that $A \alpha \leq \alpha$.

We now show that $A$ is increasing on the sector $[\beta, \alpha]$. In face, if $A v_{1}=u_{1}$ and $A v_{2}=u_{2}$, where $v_{1}, v_{2} \in[\beta, \alpha]$ and $v_{1} \leq v_{2}$. Setting $p=u_{1}-u_{2}$ we see that

$$
\begin{aligned}
& p^{\prime}=u_{1}^{\prime}-u_{2}^{\prime}=M u_{1}+f\left(t, v_{1}\right)-M v_{1}-M u_{2}-f\left(t, v_{2}\right)+M v_{2} \geq M p \text { and } \\
& p(0)=p(2 \pi) .
\end{aligned}
$$

This shows that $p \leq 0$ from Lemma 3.1. So $u_{1} \leq u_{2}$, and hence $A$ is increasing on the sector $[\beta, \alpha]$. 
On the other hand, if we define an operator $B$ on the sector $[\beta, \alpha]$ as follows:

$$
\begin{aligned}
& B v(t)=B v(0) e^{M t}+\int_{0}^{t} F(s, v(s)) e^{M(t-s)} d s \text { and } \\
& B v(0)=B v(2 \pi)=\frac{1}{e^{-2 M \pi}-1} \int_{0}^{2 \pi} F(s, v(s)) e^{-M s} d s .
\end{aligned}
$$

Then $B$ is decreasing on the sector $[\beta, \alpha]$ since $F(t, x)$ is decreasing in $\mathrm{x}$ from the conditions imposed on $f$. But obviously $B v(t)$ is a solution of PBVP (II). So operator $\mathrm{B}$ is identical with operator $A$ on the sector $[\beta, \alpha]$. And hence operator $A$ is both increasing and decreasing on the sector $[\beta, \alpha]$ and satisfies (i). From this it follows that $A$ transforms the sector $[\beta, \alpha]$ onto a point $u^{*} \in[\beta, \alpha]$. This implies that $u^{*}$ is the unique fixed point of $A$ on the sector $[\beta, \alpha]$. However solving PBVP (3.1)-(3.2) is equivalent to finding fixed points of operator $A$. Hence $u^{*}$ is the unique solution of PBVP (3.1)-(3.2) on the sector $[\beta, \alpha]$.

\section{REFERENCES}

1 V. Lakshmikantham, G.S. Ladde and A.S. Vatsala, Monotone Iterative Technique for Nonlinear Differential Equations, Pitmann, Boston, 1985.

2 V. Lakshmikantham and S. Leela, Remarks on first and second order periodic boundary value problems, Nonlinear Analysis, 8(1984), 281-287.

3 R. Kannan and V. Lakshmikantham, Existence of periodic solutions of nonlinear boundary value problems and the method of upper and lower solutions, Appl. Anal., 17(1984), 103-113.

4 V. Lakshmikantham, Periodic boundary value problems of first and second order differential equations, J. of Appl. Math. and Simulation, 2:3 (1989), 131 - 138 . 


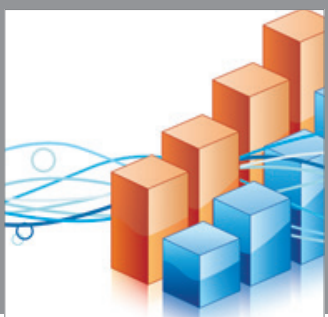

Advances in

Operations Research

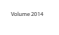

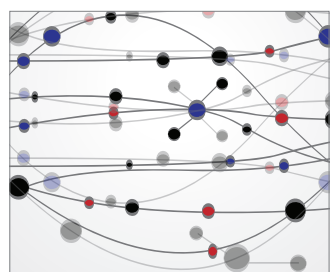

\section{The Scientific} World Journal
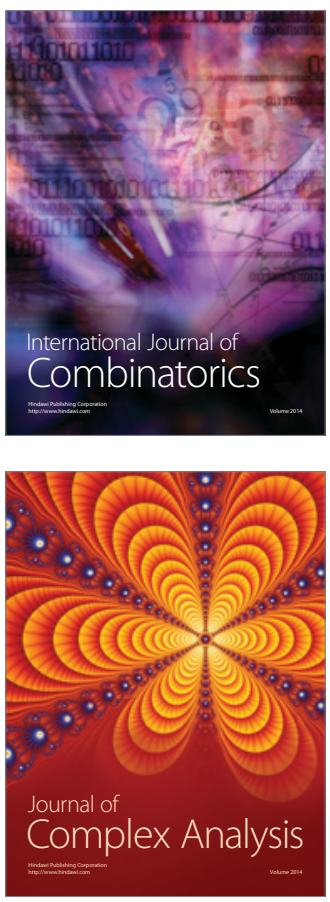

International Journal of

Mathematics and

Mathematical

Sciences
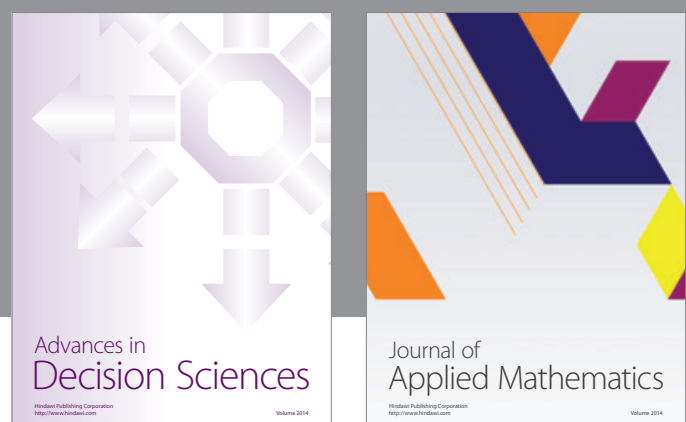

Journal of

Applied Mathematics
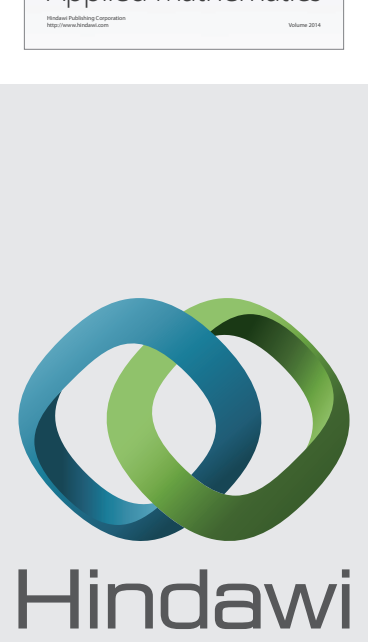

Submit your manuscripts at http://www.hindawi.com
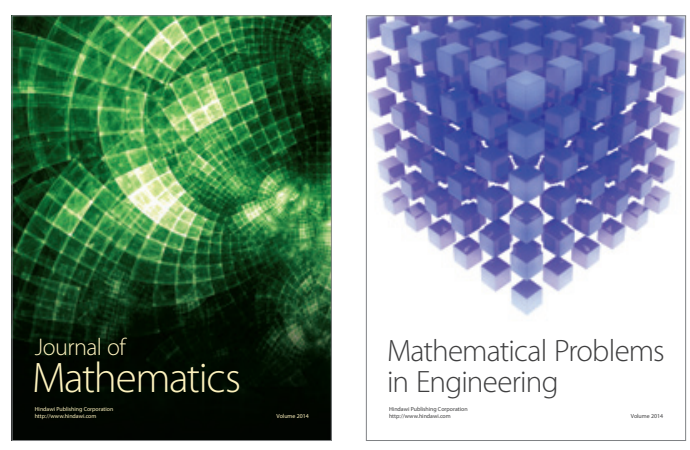

Mathematical Problems in Engineering
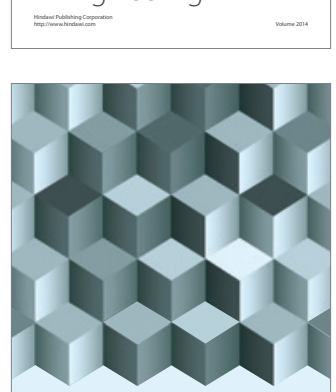

Journal of

Function Spaces
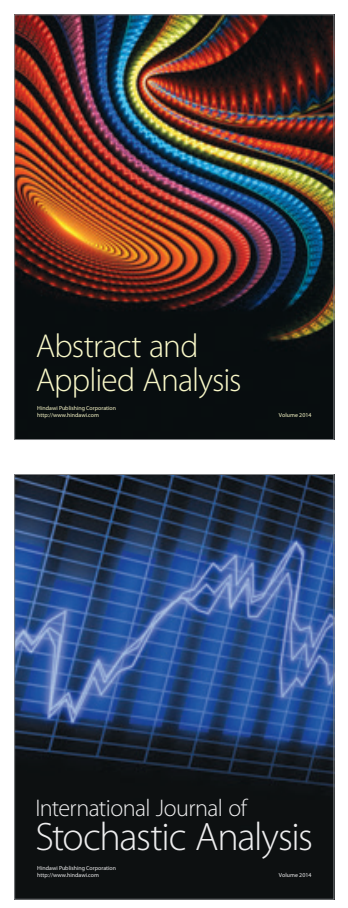

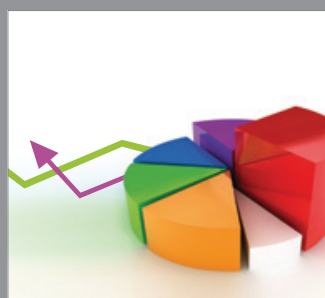

ournal of

Probability and Statistics

Promensencen
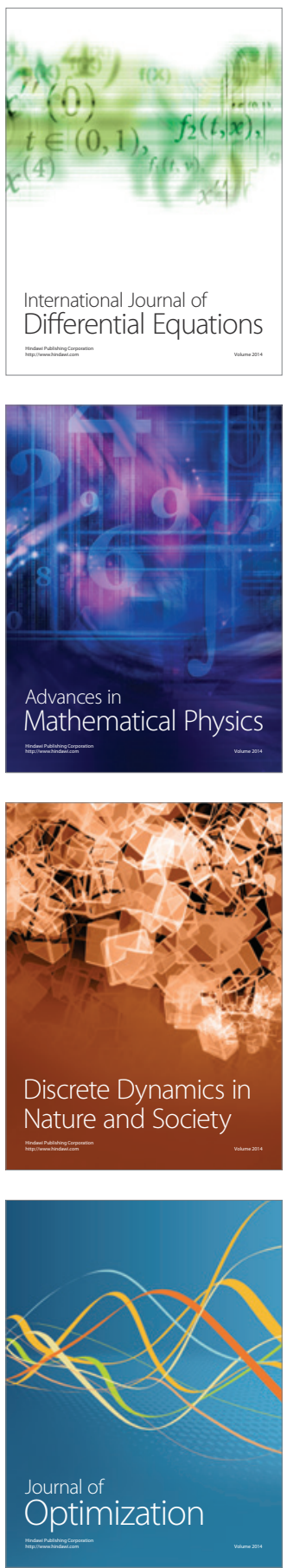